Classification

Physics Abstracts

$82.70-68.10-87.45$

\title{
Relaxation modes of an adhering bilayer membrane
}

\author{
Martin Kraus and Udo Seifert \\ Institut für Festkörperforschung, Forschungszentrum Jülich, 52425 Jülich, Germany
}

(Received 24 March 1994, accepted 7 April 1994)

\begin{abstract}
We determine the relaxational dynamics of the shape fluctuations of a fluid membrane in the vicinity of a substrate. Extending the "classical" description, we include the coupling between the local shape and the difference of the two monolayer densities as well as a lateral tension in the membrane. These extensions introduce additional length scales to the problem. The asymptotic behavior of the dispersion relation and the correlation functions can be understood from limiting cases in which either a free bilayer or a bound incompressible membrane is considered. In many cases, however, the relevant length scales do not separate very well, so that the full dispersion relation will be needed for the interpretation of experiments. It is shown that in addition to the damping due to bulk viscosity the dissipation due to friction between the monolayers is observable and indeed dominates the long-time behavior of the dynamical height correlation function for large wave vectors. As demonstrated with typical sets of parameters, the transition to this regime will be accessible by optical techniques only for weak adhesion and strong friction between the monolayers.
\end{abstract}

\section{Introduction.}

One of the earliest phenomena to receive attention in the study of the physical properties of biological membranes $[1,2]$ has been the slow relaxational dynamics of thermally excited membrane shape fluctuations, such as the "flicker" phenomenon of red blood cells [3]. Fluid phospholipid membranes, although lacking a cytoskeleton and membrane proteins, serve as simple model systems for biological membranes, and are experimentally accessible in form of giant vesicles. These vesicles also exhibit strong shape fluctuations which for free, quasispherical vesicles have been used to determine the bending rigidity, which is the essential material parameter of lipid membranes [4-6]. In addition, the dynamical correlations of these fluctuations give information about the main dissipative mechanisms.

The effect of adhesion or interaction with a substrate on these membranes can be studied in an experimental set-up recently developed by Rädler and Sackmann [7-9]. They have shown that one can obtain the static and dynamic height correlation functions of the bound part of an adhering giant vesicle using reflection interference contrast microscopy. 
In the "classical" model of a free membrane, the membrane is treated as a single, incompressible fluid sheet, whose energy is solely determined by the resistance to bending [10], and for which the damping is provided by the surrounding viscous fluid [3]. Three modifications to this classical treatment of the bending modes of a membrane are necessary to represent the experimental situation of an adhering membrane.

First of all, the presence of a substrate modifies the static fluctuation spectrum as well as the hydrodynamics $[11,12]$. Secondly, the membrane will be part of a bound vesicle. For wave-lengths much smaller than the size of the vesicle (where up to $50 \mu \mathrm{m}$ are accessible), the geometrical complications of the closed shape should not affect the dynamics. Therefore, results obtained for a bound planar membrane should apply provided the constraints on vesicle volume and area are effectively included in the description as a finite tension in the membrane $[13,14]$.

Finally, recent work on the equilibrium shapes of vesicles has demonstrated the relevance of the coupling between the local curvature and the local density of lipids within the two monolayers [15]. For static phenomena such as the equilibrium shapes of vesicles, this effect adds an additional global energy term, the area-difference-elasticity [16-19]. In the dynamics, bending is coupled to differences in the local lipid density in the monolayers, because bending the membrane will compress the inner and stretch the outer monolayer. Since the lipid monolayers are free to slide over each other, these density differences may relax $[15,20]$. Thus, the friction between monolayers will provide an additional dissipative mechanism for the damping of undulations [21]. In the small- $q$ regime, the density difference relaxation is too fast to have any effect on the height correlations. For undulations with a sufficiently large wave-vector $q$, however, the relaxation rate of the monolayer density difference, which scales as $q^{2}$, will always be slower than the relaxation of undulations, which scales as $q^{3}$ [3]. Due to the coupling between curvature and density, in this regime undulations will relax by a fast process only to the extent that they minimize the energy for given density difference. The slow decay of this density difference then dominates the long-time behavior of the height correlations.

The aim of this work is to combine these three aspects in order to explore the full dynamical behavior of a lipid bilayer membrane near a wall. In particular, we are interested in the circumstances, under which the density relaxation makes an observable contribution to the height fluctuations. One therefore needs to know not only the dispersion relation but also the time-dependent correlation function for the height variable. These quantities inevitably have a rich structure due to the presence of four length-scales. Two length-scales follow from the static correlation function in which the potential, the tension and the bending energy all scale differently with the wave-vector. As a third length-scale, the separation from the wall affects the dynamics. Finally, the fourth length-scale arises from the coupling to the density difference. Since for a bound membrane, in contrast to the free bilayer, the relaxation rate of the height correlations can become faster than the density relaxation also for small $q$, one might first expect that the double layer aspect of the membrane is then relevant for all $q$. However, we will show that the density mode will be observable in the height correlation function only if the undulation energy is dominated by the resistance to bending. For small tension, i.e. weak adhesion, the cross-over to this regime is in the visible range and the coupling to the local density difference should, indeed, have observable effects for a bound membrane.

The paper is organized as follows. In section 2, the general framework of the calculation is set up. We develop the solution of the full problem starting from limiting cases. In section 3 , we discuss the free bilayer, for which we here also calculate the time-dependent correlation functions. In section 4, we briefly review the relaxation rate for a bound membrane if the bilayer aspect is ignored. From these two limiting cases, the numerical results for the full problem can be understood. Rather than discussing the dispersion relation and the corresponding 
correlation function for all possible cases, we present these quantities for three experimentally motivated examples in section 5. Section 6 contains the conclusion. In Appendix A, the lengthy matrix entries which are the basis for the numerical calculations are given for future reference. In Appendix B, we justify the low Reynolds number approximation. Appendix $\mathrm{C}$ deals with the modifications which arise for a typical experimental situation where the fluctuations are measured within a linear strip. In Appendix D, we discuss the effect of finite slip at the substrate as well as the possibility of another slow mode occurring for multilamellar vesicles. Brief accounts of the dispersion relations for the two limiting cases have been presented in references $[11,21]$.

\section{Statics and dynamics of a bound bilayer.}

In order to calculate the relaxation modes for both height and monolayer density fluctuations, we first recall the energy of a bilayer. The lipid densities at the neutral surface of each monolayer, $\phi^{ \pm}$, have an equilibrium value $\phi_{0}$ for a flat membrane (in this paper $(+)$ will generally denote the monolayer opposite to the wall and (-) the monolayer facing the wall). It is useful to project these densities within each monolayer to a common surface, the bilayer midsurface. These projected densities, $\psi^{ \pm}$, will deviate from $\phi^{ \pm}$if the membrane is bent. With $H$ as the mean curvature of the bilayer and $d$ as the distance between the midsurface of the bilayer and the neutral surface of a monolayer, this relation reads $\phi^{ \pm} \approx \psi^{ \pm}(1 \pm 2 d H)$ to lowest order in $d H$. Expressed in terms of a scaled density deviation from the equilibrium value, $\rho^{ \pm} \equiv\left(\psi^{ \pm} / \phi_{0}-1\right)$, the elastic energy density of each monolayer, $f^{ \pm}$, is then given by

$$
f^{ \pm}=\frac{k}{2}\left(\frac{\phi^{ \pm}}{\phi_{0}}-1\right)^{2}=\frac{k}{2}\left(\rho^{ \pm} \pm 2 d H\right)^{2}\left(1+O(d H)+O\left(\rho^{ \pm}\right)\right)
$$

where $k$ is the area compression modulus of a monolayer [21].

We consider a membrane bound by a potential $V(l)$ whose minimum is at a distance $l_{0}$ from a planar wall [22]. Denoting the local displacement of the membrane by $h(x, y) \equiv l(x, y)-l_{0}$, we can express the mean curvature for small displacements as $H=\frac{1}{2} \nabla^{2} h$. The continuum free energy, $F$, for the entire membrane then is a functional of the membrane shape, $h(x, y)$, and the two densities $\rho^{ \pm}(x, y)$,

$$
F=\frac{1}{2} \int \mathrm{d} x \mathrm{~d} y\left\{\kappa\left(\nabla^{2} h\right)^{2}+\Sigma(\nabla h)^{2}+\Omega h^{2}+k\left[\left(\rho^{+}+d \nabla^{2} h\right)^{2}+\left(\rho^{-}-d \nabla^{2} h\right)^{2}\right]\right\} .
$$

The first three terms represent the energy of undulations at relaxed densities. This energy is given by the bending energy of each monolayer with the usual bilayer bending rigidity $\kappa$ [10], a tension $\Sigma$, which arises from the constraints on area and volume of the vesicle [13, 14], and the contribution from the potential, which we expand about its minimum at $l=l_{0}$ with $\Omega=\mathrm{d}^{2} V /\left.\mathrm{d} l^{2}\right|_{l=l_{0}}$. Within the range of wave-lengths accessible by optical methods, these tensions typically can become as relevant as the bending rigidity [23].

For small deviations from the planar state, we consider a plane wave in the $x$-direction, $h(x, y) \equiv h_{q} \mathrm{e}^{\imath q x}$ and $\rho^{ \pm}(x, y) \equiv\left(\bar{\rho}_{q} \pm \rho_{q}\right) \mathrm{e}^{\imath q x}$, written in terms of the difference, $\rho \equiv\left(\rho^{+}+\rho^{-}\right) / 2$, and the average, $\bar{\rho} \equiv\left(\rho^{+}-\rho^{-}\right) / 2$, of the scaled projected densities of the monolayers. In the Fourier-transformed free energy,

$$
F=\frac{1}{2}\left(h_{q}, \rho_{q}, \bar{\rho}_{q}\right) \mathbf{E}(q)\left(\begin{array}{c}
h_{q} \\
\rho_{q} \\
\bar{\rho}_{q}
\end{array}\right)^{*}
$$


where we have defined

$$
\mathbf{E}(q) \equiv\left(\begin{array}{ccc}
\tilde{\kappa} q^{4}+\Sigma q^{2}+\Omega & -2 k d q^{2} & 0 \\
-2 k d q^{2} & 2 k & 0 \\
0 & 0 & 2 k
\end{array}\right)
$$

$\bar{\rho}$ decouples from $h$ and $\rho$. The star denotes complex conjugation. Here

$$
\tilde{\kappa}=\kappa+2 d^{2} k
$$

is a renormalized bending rigidity which includes the effects of elastic deformation of the monolayers. Note that integrating out the density variables, which on the Gaussian level is equivalent to minimizing with respect to them, one recovers the undulation energy of a bound incompressible membrane with

$$
F_{0} \equiv \min _{\left\{\rho_{q}, \bar{\rho}_{q}\right\}} F=\frac{1}{2} h_{q}\left(\kappa q^{4}+\Sigma q^{2}+\Omega\right) h_{q}^{*} \equiv \frac{1}{2} h_{q} E_{0}(q) h_{q}^{*}
$$

The static correlation functions can be obtained from the inverse of the energy matrix $\mathbf{E}$, which leads to

$$
\begin{aligned}
\mathbf{G}_{0}(q) \equiv\left\langle\left(\begin{array}{c}
h_{q} \\
\rho_{q} \\
\bar{\rho}_{q}
\end{array}\right)\left(h_{q}, \rho_{q}, \bar{\rho}_{q}\right)^{*}\right\rangle_{0} & =\frac{k_{\mathrm{B}} T}{L_{x} L_{y}} \mathbf{E}^{-1}(q) \\
& =\frac{k_{\mathrm{B}} T}{L_{x} L_{y} E_{0}(q)}\left(\begin{array}{ccc}
1 & d q^{2} & 0 \\
d q^{2} & E_{h h}(q) / 2 k & 0 \\
0 & 0 & E_{0}(q) / 2 k
\end{array}\right)
\end{aligned}
$$

where $L_{x}$ and $L_{y}$ denote the linear dimensions of the rectangular patch of membrane under consideration.

For the calculation of the dynamical correlation functions, we have to consider the hydrodynamics of both the fluid bilayer and the surrounding aqueous solution. The physical properties of the membrane and its undulation energy will enter the boundary conditions and the force balance at the membrane.

The hydrodynamics of the incompressible bulk fluid is treated within the Stokes approximation,

$$
\boldsymbol{\nabla} \cdot \mathbf{v}=0 \quad \text { and } \quad \eta \nabla^{2} \mathbf{v}=\nabla p \quad \text { for } \quad z \neq l_{0} .
$$

This approximation, neglecting the inertial and advection terms of the Navier-Stokes equation, is valid for low Reynolds numbers, an assumption which has to be checked afterwards (see Appendix B).

We again assume plane waves in the $x$-direction, with velocity $\mathrm{v}=\left(v_{q, x}(z) \mathbf{e}_{x}+v_{q, z}(z) \mathbf{e}_{z}\right) \mathrm{e}^{2 q x}$ and pressure $p=p_{q}(z) \mathrm{e}^{2 q x}$ The general solution to the hydrodynamic equations (8) satisfying the condition of vanishing velocity at the wall and at infinity then is

$$
\begin{aligned}
v_{q, z}^{-}(z) & =[A(\sinh (q z)-q z \cosh (q z))+B q z \sinh (q z)] \\
p_{q}^{-}(z) & =2 \eta q[-A \cosh (q z)+B \sinh (q z)] \\
v_{q, z}^{+}(z) & =\left[C \mathrm{e}^{-q\left(z-l_{0}\right)}+D q\left(z-l_{0}\right) \mathrm{e}^{-q\left(z-l_{0}\right)}\right] \\
p_{q}^{+}(z) & =2 \eta q D \mathrm{e}^{-q\left(z-l_{0}\right)}
\end{aligned}
$$


where the indices \pm apply to $z \gtrless l_{0}$, respectively, and $v_{q, x}^{ \pm}(z)=i \partial_{z} v_{q, z}^{ \pm}(z) / q$. The constants $A, B, C$, and $D$ are to be determined by boundary conditions at the membrane, which for small displacements may be evaluated at $z=l_{0}$ : the normal velocity $v_{z}$ has to be continuous, whereas assuming a no-slip boundary condition between lipid and water means that $v_{x}^{ \pm}\left(l_{0}\right)$ has to coincide with the velocities of the lipid flow within the monolayers. Furthermore, the forces have to balance in the normal direction at the membrane, leading to

$$
-T_{z z}^{+}+T_{z z}^{-}=-\frac{\partial F}{\partial h_{q}^{*}}
$$

where we have introduced the liquid stress tensor $T_{\imath \jmath}^{ \pm} \equiv-p \delta_{\imath \jmath}+\eta\left(\partial_{i} v_{\jmath}+\partial_{\jmath} v_{\imath}\right)$, evaluated at the upper and lower monolayers. Denoting the monolayer surface pressures with $\sigma^{ \pm}$, the membrane surface viscosity with $\mu$, and the interlayer friction (whose order of magnitude can be estimated from experiments as in Refs. [24, 15]) with $b$, the lateral force balance at the two monolayers reads

$$
-\tilde{\mathbf{\nabla}} \sigma^{ \pm} \pm T_{x z}^{ \pm}+\mu \widetilde{\nabla}^{2} \widetilde{\mathbf{v}}^{ \pm} \mp b\left(\widetilde{\mathbf{v}}^{+}-\tilde{\mathbf{v}}^{-}\right)=0 .
$$

The tilde refers to two-dimensional quantities. At this point, we neglect the fact that the various forces act in different surfaces of the monolayer, e.g. the intermonolayer friction in the bilayer midplane, and the coupling to bulk water at the lipid headgroups. A more sophisticated treatment including this effect should then also incorporate orientational degrees of freedom of the lipids within the monolayer, which are excited by the torques produced in this way.

Equations (10) and (11) and the boundary conditions are sufficient to solve for all of the amplitudes $A, B, C$, and $D$ of both the flow field $\mathbf{v}(x, z)$ and the pressure $p(x, z)$ as a function of $h_{q}, \rho_{q}$ and $\bar{\rho}_{q}$. Using the assumption of impermeability of the lipid membrane to water flow [25], i.e. $\mathrm{d} h_{q} / \mathrm{d} t=v_{q, z}\left(l_{0}\right)$, and the continuity equations for the monolayer densities, $\partial_{t} \rho_{q}^{ \pm} \approx$ $-\widetilde{\nabla} \cdot \widetilde{\mathbf{v}}_{q}^{ \pm}$, which are correct when neglecting the slow lipid flip-flop between the monolayers, we obtain the relaxational dynamics in the form

$$
\frac{\mathrm{d}}{\mathrm{d} t}\left(\begin{array}{c}
h_{q} \\
\rho_{q} \\
\bar{\rho}_{q}
\end{array}\right)=-\boldsymbol{\Gamma}\left(q, l_{0}\right) \mathbf{E}(q)\left(\begin{array}{c}
h_{q} \\
\rho_{q} \\
\bar{\rho}_{q}
\end{array}\right)
$$

The explicit expression for the matrix of kinetic coefficients $\Gamma$, which together with the energy matrix $\mathbf{E}$ contains the full information about the dynamics, is given in Appendix A. Note that this matrix couples the average density $\bar{\rho}_{q}$ to both $h_{q}$ and $\rho_{q}$. We can now calculate the relaxation times as the inverse values of the eigenvalues $\gamma_{\imath}$ of $\boldsymbol{\Gamma} \cdot \mathbf{E}$. As shown in Appendix A, the dynamical correlation functions can thus be expressed in the form

$$
\begin{aligned}
\left\langle h_{q}(t) h_{q}^{*}(0)\right\rangle & =\left\langle h_{q} h_{q}^{*}\right\rangle_{0} \sum_{i=1}^{3} A_{\imath}^{h_{2}} \mathrm{e}^{-\gamma_{\imath}(q) t} \\
\left\langle\rho_{q}(t) \rho_{q}^{*}(0)\right\rangle & =\left\langle\rho_{q} \rho_{q}^{*}\right\rangle_{0} \sum_{i=1}^{3} A_{\imath}^{\rho} \mathrm{e}^{-\gamma_{\imath}(q) t}
\end{aligned}
$$

Here, $\langle. .\rangle_{0}$ are the static correlation functions given by equation (7), and the amplitudes $A_{2}^{h, \rho}$ sum up to unity, $\sum_{2} A_{2}^{h}=\sum_{2} A_{2}^{\rho}=1$, to ensure the fluctuation-dissipation-theorem. The explicit expressions for $\boldsymbol{\Gamma}$ are too complex to allow for an analytical calculation of the eigenvalues and eigenvectors of $\boldsymbol{\Gamma} \cdot \mathbf{E}$. It turns out, however, that most of the numerical results for the full problem can be understood in terms of two previously considered limiting cases $[21,11,12]$. 


\section{Free bilayer membrane.}

The case of a free bilayer membrane, which formally corresponds to $l_{0} \rightarrow \infty$, has been investigated in reference [21]. We will recall the results briefly, and, as a new result, calculate the correlation functions for this case. Due to the up-down symmetry, the fluctuations of the density average $\bar{\rho}$ decouple from $h$ and the density difference $\rho$. The matrix of kinetic coefficients for $h$ and $\rho$ is diagonal,

$$
\Gamma^{f}(q) \equiv\left(\begin{array}{cc}
1 / 4 \eta q & 0 \\
0 & \frac{q^{2}}{2\left(2 b+2 \eta q+\mu q^{2}\right)}
\end{array}\right)
$$

while the energy matrix $\mathbf{E}^{\mathbf{f}}$ is just the upper left $(2 \times 2)$ submatrix of $\mathbf{E}$ as defined in equation (4).

The product $\Gamma^{\mathrm{f}}(q) \mathbf{E}^{\mathrm{f}}(q)$ has two eigenvalues $\gamma_{1,2}^{\mathrm{f}}(q)$, which yield the dispersion relations shown in figure 1a. For simplicity, in this section, we discuss only the case of vanishing tension. There are three regimes [21], separated by the crossover wavevectors $q_{1}^{\mathrm{f}} \equiv 2 \eta k / b \kappa[26]$ and $q_{2}^{\mathrm{f}} \equiv \sqrt{2 b / \mu}$

$$
\gamma_{1}^{\mathrm{f}} \approx\left\{\begin{array} { c c } 
{ \frac { \kappa } { 4 \eta } q ^ { 3 } , } & { q \ll q _ { 1 } ^ { \mathrm { f } } } \\
{ \frac { k } { 2 b } \frac { \kappa } { \tilde { \kappa } } q ^ { 2 } , } & { q _ { 1 } ^ { \mathrm { f } } \ll q \ll q _ { 2 } ^ { \mathrm { f } } } \\
{ \frac { k } { \mu } \frac { \kappa } { \tilde { \kappa } } , } & { q _ { 2 } ^ { \mathrm { f } } \ll q }
\end{array} \quad \gamma _ { 2 } ^ { \mathrm { f } } \approx \left\{\begin{array}{cc}
\frac{k}{2 b} q^{2}, & q \ll q_{1}^{\mathrm{f}} \\
\frac{\tilde{\kappa}}{4 \eta} q^{3}, & q_{1}^{\mathrm{f}} \ll q .
\end{array}\right.\right.
$$

The amplitudes $A_{2}^{h}$ and $A_{2}^{\rho}$ of the contribution of the two modes to the correlation functions $\left\langle h_{q}(t) h_{q}(0)\right\rangle$ and $\left\langle\rho_{q}(t) \rho_{q}(0)\right\rangle$ (see Eqs. (13), (14)), as calculated numerically, are shown in figure $1 \mathrm{~b}$.

For $q \ll q_{1}^{\mathrm{f}}$, there is the "classical" bending mode of a free membrane damped by bulk viscosity, as given by $\gamma_{1}^{\mathbf{f}}$, as well as a second mode due to interlayer friction, which decays fast. The asymptotic behavior of the correlation functions can be derived as follows. Undulations relax only by the slow mode, so $A_{1}^{h} \approx 1$, and $A_{2}^{h}$ is negligibly small, since the height variable is too slow to follow the fast density fluctuations. Density differences between the monolayers, however, relax by both modes. On time scales longer than $1 / \gamma_{2}^{\mathrm{f}}$, the density relaxes as to minimize the free energy for given undulations. This condition, $\partial F /\left.\partial \rho_{q}^{*}\right|_{h_{q}}=0$, implies $\rho_{q}=$ $d q^{2} h_{q}$, and therefore

$$
\left\langle\rho_{q}(t) \rho_{q}^{*}(0)\right\rangle \approx d^{2} q^{4}\left\langle h_{q}(t) h_{q}^{*}(0)\right\rangle \approx \frac{k_{\mathrm{B}} T d^{2}}{L_{x} L_{y} \kappa} \mathrm{e}^{-\gamma_{\mathrm{I}}^{\mathrm{f}} t}=\left\langle\rho \rho^{*}\right\rangle_{0} \frac{2 k d^{2}}{\tilde{\kappa}} \mathrm{e}^{-\gamma_{\mathrm{i}}^{\mathrm{f}} t}
$$

From this relation and equation (14) we obtain the coefficient $A_{1}^{\rho} \approx 2 k d^{2} / \tilde{\kappa}$ for long times $t \gg 1 / \gamma_{2}^{\mathrm{f}}$, for which it is determined solely by the coupling to height fluctuations. Consequently, $A_{2}^{\rho} \approx 1-A_{1}^{\rho}=\kappa / \tilde{\kappa}$, so that the ratio of the amplitudes of both modes in the correlation function is given by the ratio of the two contributions to the renormalized bending rigidity (5).

At $q_{1}^{\mathrm{f}}$ the two damping rates become comparable, so that for $q \gg q_{1}^{\mathrm{f}}$ the density relaxation is the slowest mode. Density fluctuations will therefore not be induced by the quickly decaying undulations, so that $A_{1}^{\rho} \approx 1$. Fast height fluctuations take place at unrelaxed density difference, which leads to a renormalization of the bending rigidity from $\kappa$ to $\tilde{\kappa}$ for the faster mode $\gamma_{2}^{\mathrm{f}}$. Height fluctuations induced by density differences relax quickly to $h_{q}=2 k d \rho_{q} / \tilde{\kappa} q^{2}$ (as can 


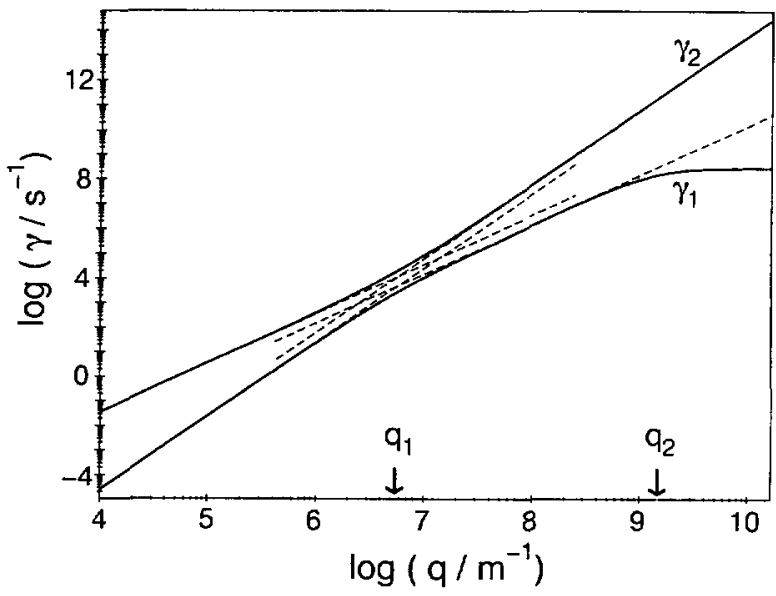

(a)

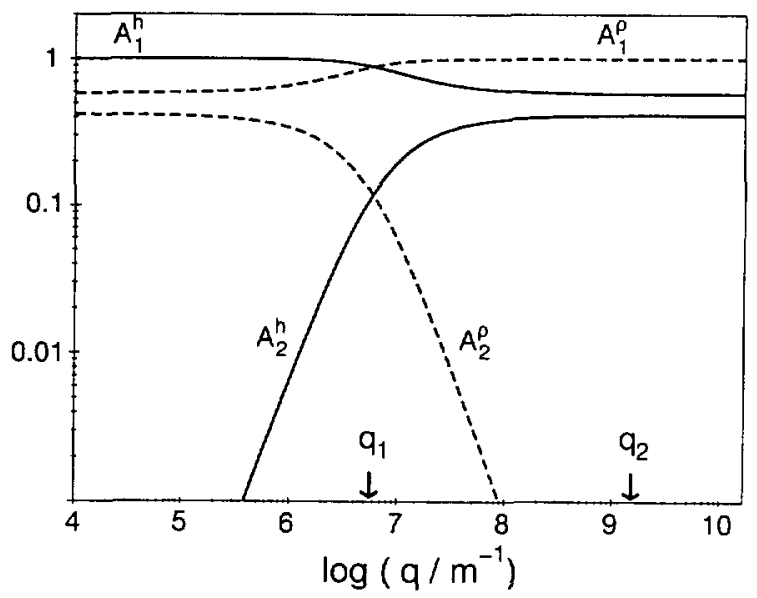

(b)

Fig. 1. - (a) Dispersion relations for a free bilayer for $\kappa=10^{-19} \mathrm{~J}, k=0.07 \mathrm{~J} / \mathrm{m}^{2}, d=1 \mathrm{~nm}$, $\eta=10^{-3} \mathrm{~J} \mathrm{~s} / \mathrm{m}^{3}, \mu=10^{-10} \mathrm{~J} \mathrm{~s} / \mathrm{m}^{2}$, and $b=10^{8} \mathrm{~J} \mathrm{~s} / \mathrm{m}^{4}$; the dashed lines indicate the asymptotic behavior $\gamma_{1} \approx \kappa q^{3} / 4 \eta$ and $\gamma_{2} \approx k q^{2} / 2 b$, for small $q_{;} \gamma_{2} \approx \tilde{\kappa} q^{3} / 4 \eta$ and $\gamma_{1} \approx k q^{2} \kappa / 2 b \tilde{\kappa}$, for large $q$, respectively. (b) Amplitudes of the dynamic correlation functions for a free bilayer as defined in equations $(13,14)$. The ratio $\kappa / \tilde{\kappa} \simeq 0.417$ determines the asymptotic behavior of both the amplitudes $A_{2}^{\rho}$ for small $q$ and $A_{2}^{h}$ for large $q$.

be calculated from $\partial F /\left.\partial h_{q}^{*}\right|_{\rho_{q}}=0$ ), which yields $A_{1}^{h} \approx 2 d^{2} k / \tilde{\kappa}$, similar to the calculation displayed in equation (17).

Finally, for $q \gg q_{2}^{\mathrm{f}}$ interlayer friction is replaced by monolayer surface viscosity as the dominant in-membrane damping mechanism in $\gamma_{1}^{\mathrm{f}}[27]$. This crossover, however, does not affect the amplitudes in the correlation function.

An important consequence of these results is that any experiment measuring $\left\langle h_{q}(t) h_{q}(0)\right\rangle$ will also pick up a contribution from the slow density mode for $q>q_{1}^{\mathrm{f}}$, provided it is sensitive to the time-scale $1 / \gamma_{1}^{f}$. In particular, the slowest time-scale in this regime will scale as $q^{-2}$ rather than as $q^{-3}$ In fact, recent measurements of the undulation modes on lipid multilayers using a neutron spin-echo method can be interpreted in this way [28]. For $q \ll q_{1}^{f}$, however, the fast membrane-damped mode is not visible in the height correlations. 


\section{Bound membrane without internal modes.}

Neglecting the bilayer aspect and the in-plane density modes one finds the relaxational behavior of a bound incompressible membrane as calculated in references [11, 12]. This limit can also be obtained analytically from the full solution as obtained with the formalism of section 2 . The damping rate, $\gamma_{s}$, of the single mode can be split in a product of a dynamical part $\Gamma^{\mathbf{s}}$ and the energy $E_{0}$ (cf. Eq. (6)),

$$
\gamma^{\mathbf{s}}\left(q, l_{0}, \xi_{\Sigma}, \xi_{\kappa}\right)=\Gamma^{\mathrm{s}}\left(q, l_{0}\right) E_{0}\left(q, \xi_{\Sigma}, \xi_{\kappa}\right)=\Gamma^{\mathrm{s}}\left(q, l_{0}\right) \Sigma\left[\xi_{\kappa}^{-2} q^{4}+q^{2}+\xi_{\Sigma}^{-2}\right] .
$$

For wave-vectors below $q=\xi_{\Sigma}^{-1} \equiv \sqrt{\Omega / \Sigma}$ the dominant contribution to the undulation energy arises from the potential. Above $q=\xi_{\Sigma}^{-1}$ tension dominates the potential, until bending energy dominates above $q=\xi_{\kappa}^{-1} \equiv \sqrt{\Sigma / \kappa}$. If the tension is too small to produce a tensiondominated regime, i.e. for $\Sigma<\sqrt{\Omega \kappa}$, there is only one crossover at $q=\xi^{-1}$ with $\xi \equiv(\Omega / \kappa)^{1 / 4}$.

The hydrodynamical flow fields fall of as $q^{-1}$ perpendicular to the membrane, cf. equation (9). Consequently, for $q<l^{-1}$ the presence of the wall modifies the damping. This crossover shows up in the kinetic coefficient

$$
\begin{aligned}
\Gamma^{s}\left(q, l_{0}\right) & \equiv \frac{1}{2 \eta q} \frac{\sinh ^{2}\left(q l_{0}\right)-\left(q l_{0}\right)^{2}}{\sinh ^{2}\left(q l_{0}\right)-\left(q l_{0}\right)^{2}+\sinh \left(q l_{0}\right) \cosh \left(q l_{0}\right)+\left(q l_{0}\right)} \\
& \approx\left\{\begin{array}{cc}
l_{0}^{3} q^{2} / 12 \eta, & q \ll 1 / l_{0} \\
1 / 4 \eta q, & q \gg 1 / l_{0},
\end{array}\right.
\end{aligned}
$$

where the $q^{2}$-dependence for small $q$ can be understood as the effect of conserving the enclosed fluid volume.

Depending on the relative size of the crossover length-scales $l_{0}, \xi_{\Sigma}$, and $\xi_{\kappa}$, different cases have to be distinguished for the $q$-dependence of the damping rate $\gamma^{\mathbf{s}}$. For $q \ll \min \left\{l^{-1}, \xi_{\Sigma}^{-1}, \xi_{\kappa}^{-1}\right\}$, one finds

$$
\gamma^{s} \approx \frac{\Omega l_{0}^{3} q^{2}}{12 \eta}
$$

The asymptotic behavior for large $q$ is that of a free membrane with $\gamma^{s} \approx \kappa q^{3} / 4 \eta$. In the intermediate regime, the damping rate depends on the relative magnitude of $l_{0}, \xi_{\Sigma}$ and $\xi_{\kappa}$. The asymptotic behavior in the various regimes can then be obtained by multiplication of the respective dominant terms of $\Gamma^{\mathbf{s}}$ and $E_{0}$.

\section{Results for the bound bilayer.}

In the full problem, the presence of the wall breaks the symmetry between $\rho^{+}$and $\rho^{-}$Therefore, the dynamics couples both variables $\rho$ and $\bar{\rho}$ to the fluctuations of $h$, which leads to three coupled modes. As will be discussed in Appendix B, however, the coupling to the $\bar{\rho}$-mode is typically weak, which allows to discuss the relaxation times and correlation functions for $h$ and $\rho$ as if they were decoupled from $\bar{\rho}$. The remaining two modes are those already discussed above: a hydrodynamic mode arising from the damping of membrane undulations by bulk viscosity and a density mode damped by in-membrane friction.

Due to the number of length-scales involved, there are now many different scenarii for the relaxation rates. Instead of an exhaustive discussion of all possible cases we will discuss the general behavior in terms of the mechanisms explained in the preceding sections for the two limit cases, and present the dispersion relations for three exemplary sets of parameters. 
A useful distinction arises from the criterion whether or not there is a crossover vector $q_{1}$ separating a small- $q$ regime, where the density adjusts to the height fluctuations, from a large- $q$ regime, where the density cannot follow the fast height fluctuations, whereas the height adjusts to density fluctuations. In analogy to the free bilayer discussed in section 3 , such a crossover vector $q_{1}$ would be given by the implicit equation

$$
\gamma^{\mathbf{s}}\left(q_{1}\right)=\frac{k q_{1}^{2}}{2 b}
$$

Since $\gamma^{\text {s }}$ (cf. Eq. (18)) follows regimes not present for the free bilayer, $q_{1}$ will in general differ from the value $q_{1}^{\mathrm{f}}$. A crossover vector $q_{1}$ exists, if the criterion

$$
\Omega l_{0}^{3}<6 k \eta / b
$$

is fulfilled, as follows from the small- $q$ behavior of $\gamma_{\mathrm{s}}(21)$.

For any given potential $V(l)$, this criterion can, in principle, be checked. However, it is known from renormalization group calculations on the interaction of membranes with substrates that fluctuations can renormalize such a potential [1,22], which makes the direct evaluation of criterion (23) difficult. A crude estimate can be obtained as follows. The free parameters on the right side of this criterion are independent of the potential. Typical values are $k=0.07$ $\mathrm{J} / \mathrm{m}^{2}, \eta=10^{-3} \mathrm{~J} \mathrm{~s} / \mathrm{m}^{3}, b=10^{8} \mathrm{~J} \mathrm{~s} / \mathrm{m}^{4}$. Since $\Omega l_{0}^{2}$ amounts to an adhesion energy $V_{0}$, relation (23) becomes $V_{0} l_{0}<10^{-12} \mathrm{~J} / \mathrm{m}$. With typical adhesion energies below $10^{-5} \mathrm{~J} / \mathrm{m}^{2}$, and substrate distances below $100 \mathrm{~nm}[29,9]$ this criterion will thus be met in most physical situations.

We will now discuss the dispersion relations and the correlation functions for three parameter sets. For the first two, the criterion (23) is fulfilled; in the third example we present a case where it is violated.

5.1 First example: SMALl Tension. - A first example is shown in figure 2: parameters for this case are chosen to follow an experimental example [9]. We consider a giant vesicle in weak adhesion with the fluctuating part facing the wall in an intermediate distance $l_{0} \simeq 50 \mathrm{~nm}$. Tension in such a case is small. The curvature of the potential can be estimated via $\Omega=\kappa \xi^{-4}$ (or $\Omega=\Sigma \xi^{-2}$ ) from the measured real-space correlation length of height fluctuations, which is of the order $0.5 \mu \mathrm{m}$. The remaining material parameters as given in the figure caption are chosen as for the free bilayer case.

For $q \ll q_{1}$, the density difference relaxes quickly and thus follows the undulations. The damping rate for the undulations, as given by $\gamma_{1}$, runs with increasing $q$ through the crossovers $\xi_{\Sigma}^{-1}$ and $\xi_{\kappa}^{-1}$ already present for a single layer near a substrate, discussed in section 4 . Due to the coupling of height and density, the undulations which relax slowly with damping rate $\gamma_{1}$ induce density correlations. These can be calculated analogously to equation (17) with the result

$$
A_{1}^{\rho} \approx \frac{2 k d^{2} q^{4}}{\tilde{\kappa} q^{4}+\Sigma q^{2}+\Omega} \quad\left(q \ll q_{1}\right) .
$$

So, for $q \ll \xi_{\kappa}^{-1}, A_{1}^{\rho}$ effectively vanishes, and $A_{2}^{\rho} \approx 1$, whereas at wave vectors $\xi_{\kappa}^{-1}<q<q_{1}$, one observes the low $q$ behavior of the free bilayer without tension.

The value of $q_{1} \simeq\left(6 \mathrm{k \eta} / b \kappa l^{3}\right)^{1 / 4}$ is close to $l^{-1}$ in this example. Thus for $q \gg q_{1}$ one recovers the behavior at $q \gg q_{1}^{\mathrm{f}}$ of the free bilayer as in section 3. In particular, the relaxation of density differences is too slow to follow the undulations and therefore the bending rigidity is 


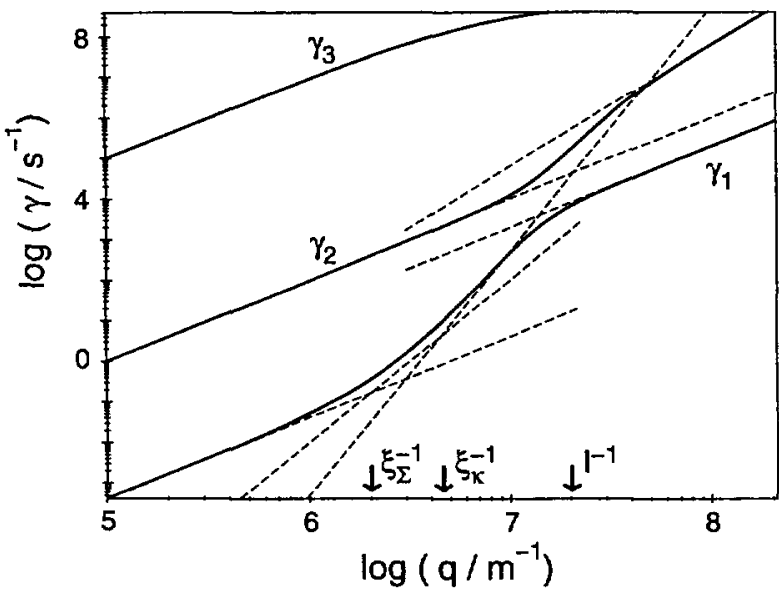

(a)

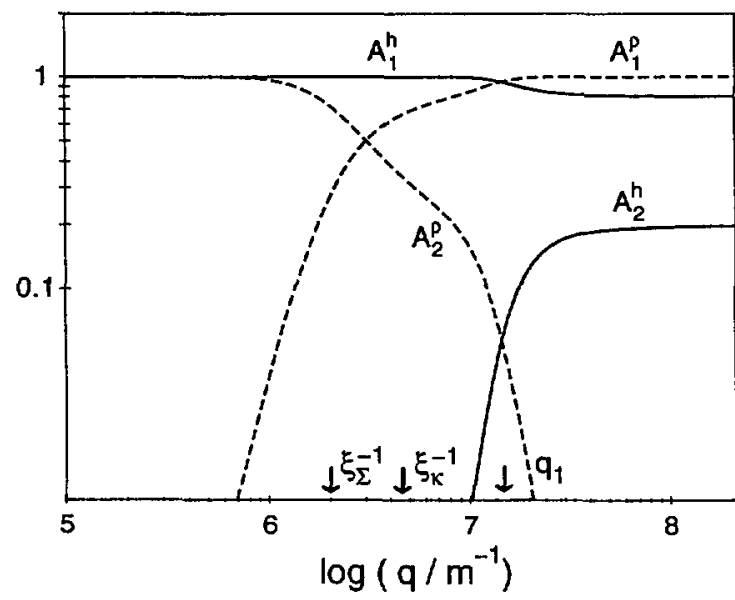

(b)

Fig. 2. - (a) Dispersion relations for a bound bilayer; case of weak adhesion and low tension. $\kappa=$ $0.5 \times 10^{-19} \mathrm{~J}, k=0.1 \mathrm{~J} / \mathrm{m}^{2}, d=1 \mathrm{~nm}, \eta=10^{-3} \mathrm{~J} \mathrm{~s} / \mathrm{m}^{3}, \mu=10^{-10} \mathrm{~J} \mathrm{~s} / \mathrm{m}^{2}, b=5 \times 10^{8} \mathrm{~J} \mathrm{~s} / \mathrm{m}^{4}$, $\Omega=4 \times 10^{6} \mathrm{~J}, \Sigma=10^{-6} \mathrm{~J} / \mathrm{m}^{2}$, and $l_{0}=50 \mathrm{~nm}$; The dashed lines indicate the asymptotic behavior in the various regimes, i.e. $k q^{2} / 2 b$ and $\tilde{\kappa} q^{3} / 4 \eta$ for $\gamma_{2} ; \Omega l^{3} q^{2} / 12 \eta, \Sigma l^{3} q^{4} / 12 \eta, \kappa l^{3} q^{6} / 12 \eta$, and $k q^{2} \kappa / 2 b \tilde{\kappa}$ for $\gamma_{1}$. Evaluation of $q_{1}$ using the full damping rate from equation (18) gives $q_{1}=1.45 \times 10^{7} \mathrm{~m}^{-1}$. The relaxation mode $\gamma_{3}$ of the average monolayer density $\bar{\rho}$ decays much faster. (b) Amplitudes of the dynamic correlation functions for a bound bilayer as defined in equations $(13,14)$. Parameters used here give $\kappa / \tilde{\kappa} \simeq 0.2$ for the large- $q$ behavior of $A_{2}^{h}$.

renormalized. As above, it is possible to calculate the asymptotics of the amplitudes of the dynamic correlation function in this regime, giving $A_{1}^{\rho} \approx 1$,

$$
A_{1}^{h} \approx \frac{2 k d^{2} q^{4}}{\tilde{\kappa} q^{4}+\Sigma q^{2}+\Omega} \quad\left(q \gg q_{1}\right),
$$

and $A_{2}^{h} \approx 1-A_{1}^{h}$. For $q \gg \xi_{\kappa}^{-1}$, the amplitude $A_{1}^{h}$ reduces to $2 k d^{2} / \tilde{\kappa}$.

5.2 Second EXAmple: LARge tension. - A second example, also motivated by an experimental situation, is presented in figure 3. Here, we consider a pancake-like vesicle which is strongly bound to the substrate. The upper side of such a vesicle is flickering, while the 


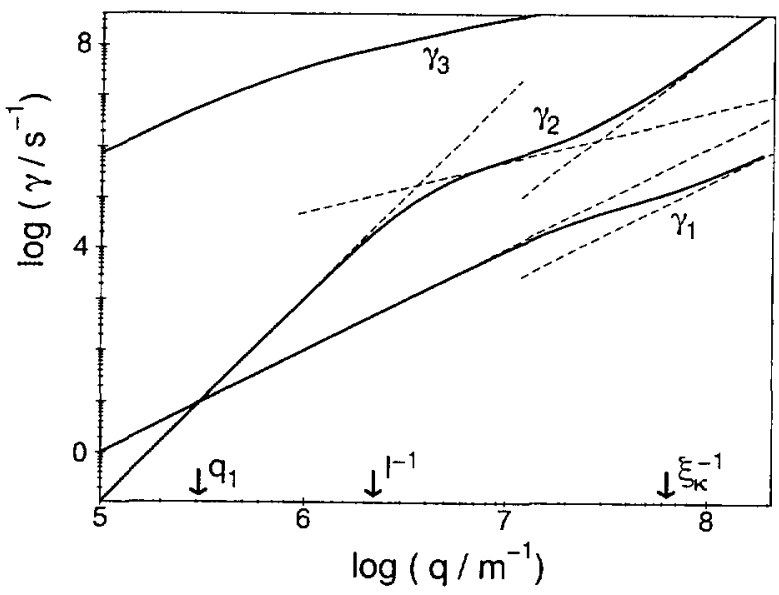

(a)

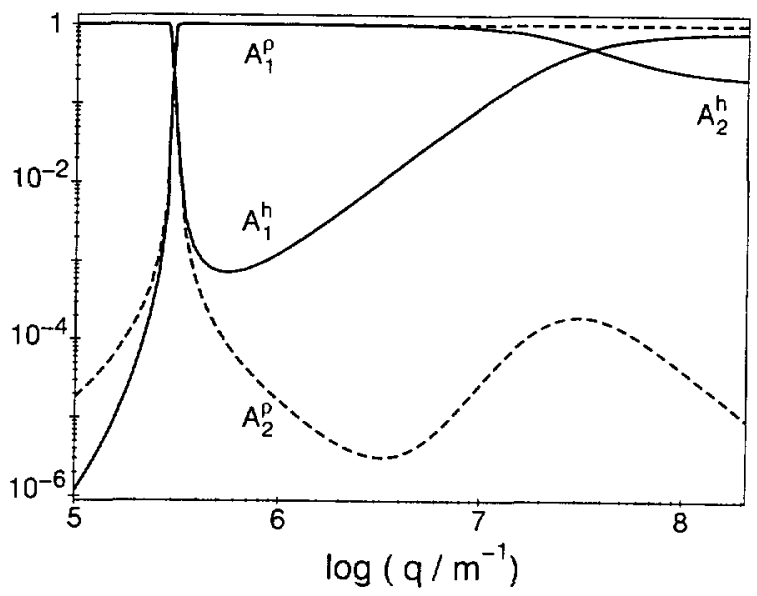

(b)

Fig. 3. - (a) Dispersion relations for a bound bilayer; case of large tension. Parameters as in figure 2, except for $\Sigma=2 \times 10^{-4} \mathrm{~J} / \mathrm{m}^{2}, l_{0}=400 \mathrm{~nm}, \Omega=0$. The dashed lines indicate the asymptotic behavior in the various regimes as discussed in the text: the in-membrane damping rate changes from $k q^{2} / 2 b$ to $k q^{2} \kappa / 2 b \bar{\kappa}$, while the bulk damping passes the asymptotical behaviors $\Sigma l^{3} q^{4} / 12 \eta, \Sigma q / 4 \eta$, and $\tilde{\kappa} q^{3} / 4 \eta$. (b) Amplitudes of the dynamic correlation functions for a bound bilayer as defined in equations (13, 14). The ratio $\kappa / \bar{\kappa}$ is as in figure 2 , whereas $A_{1}^{h}$ is determined by the coupling to density and follows equation (25) for $q \gg q_{1}$.

lower side is essentially fixed by the strong adhesion. We assume that the no-slip boundary condition at the wall, which now is coated by the fixed bilayer, is still valid (for a modification see Appendix D). Due to the large distance from the wall, the fluctuating membrane will not be held in position by a potential, but rather by the constraints on area and volume of the vesicle. Thus $\Omega$ may be neglected. Because of the strong adhesion, tension is large, but should be still one order of magnitude below the lysis tension, which is of the order $10^{-3} \mathrm{~J} / \mathrm{m}^{2}[30]$.

With parameters accordingly chosen as given in the figure caption, $q_{1}=\sqrt{6 \mathrm{k \eta} / \mathrm{b \Sigma l_{0 } ^ { 3 }}}$ turns out to be very small, and the length scales $q_{1}, l_{0}^{-1}$, and $\xi_{\kappa}^{-1}$ are well separated. For $q \ll q_{1}$, $A_{1}^{h} \simeq A_{2}^{\rho} \simeq 1$, since the coupling of density correlations to the slowly decaying undulations follows equation (24) and thus effectively vanishes for $q \ll \xi_{\kappa}^{-1}$

For $q \gg q_{1}$ there is a large tension-dominated regime, where the damping changes from 
$\gamma_{2} \approx \Sigma l^{3} q^{4} / 12 \eta$ to $\gamma_{2} \approx \Sigma q / 4 \eta$ at $q=l^{-1}$ The height correlations induced by the slow density mode follow equation (25), and thus are negligible for $q \ll \xi_{\kappa}^{-1}$ Only above $q=\sqrt{\Sigma / \tilde{\kappa}}$ does the (for all $q>q_{1}$ renormalized) bending rigidity dominate the undulation energy. From the form of $A_{1}^{h}$, (Eq. (25)), it can be seen, that only in this regime the height correlations are influenced by the slow density mode, whose damping rate $\gamma_{1}$ is then shifted by a factor $\kappa / \tilde{\kappa}$. Thus, in this example observable effects of the renormalization of bending rigidity set in at a larger value of $q$ than where the interchange of slow and fast mode takes place, contrary to the free bilayer case.

5.3 Third example: SLOW Density Relaxation for all $q$. - If the condition (23) is violated, the density relaxation is always slow and there is no crossover wave-vector $q_{1}$. Density differences cannot be induced by fast height fluctuations, resulting in a negligible $A_{2}^{\rho}$. The height correlations induced by the coupling to the slowly decaying density mode, as expressed in $A_{1}^{h}$, are now given by equation (25) for all $q$. As we have seen in the second example, however, this turns out to be an observable effect in the height correlation function, i.e. $A_{1}^{h}$ is of order unity, only for $q \gg \sqrt{\Sigma / \tilde{\kappa}}$.

As an example, we consider a charged membrane pushed by a linear potential (e.g. arising from an osmotic pressure) towards the substrate. In weak electrolytes, where the screening length is large compared to $l_{0}$, fluctuations beyond the harmonic level can safely be ignored. The potential then reads $V(l)=\pi k_{\mathrm{B}} T /\left(2 l_{\mathrm{B}} l\right)+p l$, where $l_{\mathrm{B}} \simeq 0.7 \mathrm{~nm}$ is the Bjerrum length in water. For this potential, $\Omega l_{0}^{3}=\pi k_{\mathrm{B}} T / l_{\mathrm{B}} \simeq 1.8 \times 10^{-11} \mathrm{~J} / \mathrm{m}$ is independent of $l_{0}$ and indeed violates the criterion (23).

For the relaxation rates, a large $\Omega$ means that there will be no tension-dominated regime. The two modes follow the small- $q$-behavior of $\gamma_{\mathrm{s}} \approx \Omega l_{0}^{3} q^{2} / 12 \eta(21)$ and $\gamma_{2}^{\mathrm{f}} \approx k q^{2} / 2 b(16)$, until at $q \gg\left\{\xi^{-1} \equiv(\Omega / \kappa)^{(1 / 4)}, l^{-1}\right\}$ the large- $q$ behavior of the free bilayer is recovered again.

\section{Conclusion.}

We have calculated the relaxation modes for a bound membrane with additional degrees of freedom due to monolayer density fluctuations employing linearized hydrodynamics. The presence of the wall and the undulation energy contributions by potential and tension give rise to a number of cross-over length-scales and affect not only the damping of undulations, but also the relevance of the coupling of bending and density modes.

The coupling between density and height variables becomes relevant to the height correlation function beyond a cross-over $q$-vector given by the maximum of $q_{1}$ (as defined in Eq.(22)), $1 / \xi$ and $1 / \xi_{\kappa}$ (as defined in Sect. 4 ). Since this cross-over typically is larger than the corresponding value of a free bilayer membrane, the coupling between shape and density will be more difficult to detect for a bound membrane. For typical values, as used in the examples discussed above, this cross-over corresponds to a wave-length which is at the lower end of the optically accessible range (compare Fig. 2) or even smaller as in figure 3.

If the coupling between shape and density can be neglected, the correlation function for the height variable is given by

$$
\left\langle h_{q}(t) h_{q}^{*}(0)\right\rangle=\frac{k_{\mathrm{B}} T}{L_{x} L_{y}\left(\kappa q^{4}+\Sigma q^{2}+\Omega\right)} \mathrm{e}^{-\gamma_{\mathrm{s}}(q) t},
$$

with $\gamma_{\mathrm{s}}(q)$ defined in equation (18). Since the tension in the membrane can cause a crossover in the optical range as it happens for weak adhesion shown in figure 2, the relaxation rate does not obey a simple power law for a large $q$-range. In such a case, a fit against the expression (26) is necessary. This expression contains in $\gamma_{\mathbf{s}}(q)$ two more parameters, namely the viscosity $\eta$ 
and the separation $l_{0}$, than the corresponding static correlation functions, i.e. the mean square amplitudes as given by the preexponential factor in (26). Since the viscosity is known, the dynamical measurements could be used to obtain the separation $l_{0}$ from these data. Likewise, if this separation is measured independently, the dynamical data provide a much better basis for a determination of the curvature $\Omega$ of the adhesion potential and the effective tension $\Sigma$.

In any precise determination of these quantities from experimental data, one has to be aware of additional modifications some of which we discuss in Appendices C and D. In Appendix C, we present the correlation functions as appropriate if data have been obtained only for a linear strip. As shown in Appendix D, the relaxation rate will also be slightly modified if the substrate is coated with another bilayer. There we also point out that for multilamellar vesicles a peristaltic mode causes another slow time-scale. As a consequence of all these complications, a quantitative comparison with experimental data available so far [9] has not yet been achieved.

The full analysis presented in this paper will be mandatory for the interpretation of any experiment in the cross-over regime where the height correlation function will show bi-exponential behavior. In the two examples presented here, the two time-scales are well separated even in the cross-over region but different parameter sets may lead to a closer gap between the two modes.

There are, in principle, two possibilities to reach this cross-over regime where the coupling between shape and density becomes relevant. For weak adhesion, which implies weak tension, as shown in figure 2 , the cross-over is dominated by $q_{1}$, i.e. the value of the friction coefficient $b$. If the friction is increased, the cross-over would shift towards longer wave-length. One may speculate whether close to the main transition this friction becomes larger due to the freezing of the chains. Likewise in mixtures of lipids with different chain lengths, this friction may be enhanced. Secondly, one could contemplate grazing incidence neutron reflection or, at the present stage even more speculatively, X-ray microscopy, to gain access to wave-vectors beyond $10^{7} / \mathrm{m}$. In fact, the recent analysis of a neutron spin echo experiment [28] on a stack of membranes has given strong indication for the relevance of the coupling between height and density difference for $q \simeq 10^{8}-10^{9} / \mathrm{m}$.

\section{Acknowledgments.}

We acknowledge stimulating discussions with W. Fenzl, R. Lipowsky, J. Rädler, E. Sackmann and H. Strey.

\section{Appendix A.}

\section{Explicit expression for $\boldsymbol{\Gamma}$ and calculation of modes.}

The matrix $\Gamma$ as defined in equation (12) has been calculated analytically using the computer algebra system Maple [31]. The result for the matrix elements then reads

$$
\begin{aligned}
\boldsymbol{\Gamma}_{11}= & -\left(-2 \mu b q \cosh ^{2}\left(l_{0} q\right)-2 \eta \mu q^{2} \cosh \left(l_{0} q\right) \sinh \left(l_{0} q\right)-2 \eta \mu q^{2} \cosh ^{2}\left(l_{0} q\right)\right. \\
& -\mu^{2} q^{3} \cosh ^{2}\left(l_{0} q\right)+2 \eta \mu l_{0}^{2} q^{4}+2 \eta \mu l_{0} q^{3}+4 \eta^{2} q^{2} l_{0}+\mu^{2} l_{0}^{2} q^{5}+2 b \eta l_{0} q+2 b \eta l_{0}^{2} q^{2} \\
& +2 \mu b l_{0}^{2} q^{3}+2 \eta b+\mu^{2} q^{3}+2 \eta \mu q^{2}+2 b \mu q-2 b \eta \cosh ^{2}\left(l_{0} q\right) \\
& \left.-4 \eta^{2} q \sinh \left(l_{0} q\right) \cosh \left(l_{0} q\right)-2 b \eta \cosh \left(l_{0} q\right) \sinh \left(l_{0} q\right)\right) /\left(\eta q Y_{1}\right) \\
\boldsymbol{\Gamma}_{12}= & \boldsymbol{\Gamma}_{21}=-(2 \eta+\mu q) q^{3} l_{0}^{2} / Y_{1} \\
\boldsymbol{\Gamma}_{13}= & \boldsymbol{\Gamma}_{31}=\left(2 b+2 \eta q+\mu q^{2}\right) q^{2} l_{0}^{2}\left(\cosh \left(l_{0} q\right)-\sinh \left(l_{0} q\right)\right) / Y_{2}
\end{aligned}
$$




$$
\begin{aligned}
\boldsymbol{\Gamma}_{22}= & -q^{2}\left(-\mu l_{0} q^{2} \sinh \left(l_{0} q\right)-\mu l_{0}^{2} q^{3} \cosh \left(l_{0} q\right)^{2}+\mu l_{0} q^{2} \cosh \left(l_{0} q\right)+\mu l_{0}^{2} q^{3} \sinh \left(l_{0} q\right)\right. \\
& \left.+\eta \cosh \left(l_{0} q\right)+\mu q \sinh \left(l_{0} q\right)+\eta \sinh \left(l_{0} q\right)\right) / Y_{2} \\
\boldsymbol{\Gamma}_{23}= & \boldsymbol{\Gamma}_{32}=\eta q^{2}\left(1+2 l_{0}^{2} q^{2}-2 l_{0} q\right)\left(\cosh \left(l_{0} q\right)-\sinh \left(l_{0} q\right)\right) / Y_{2} \\
\boldsymbol{\Gamma}_{33}= & -q\left(\left(\eta q+\mu l_{0}^{2} q^{4}+\mu q^{2}+2 b l_{0}^{2} q^{2}+2 b-2 b l_{0} q-\mu l_{0} q^{3}\right) \sinh \left(l_{0} q\right)\right. \\
& \left.+\left(\eta q+\mu l_{0} q^{3}-2 b l_{0}^{2} q^{2}-\mu l_{0}^{2} q^{4}+2 b l_{0} q\right) \cosh \left(l_{0} q\right)\right) / Y_{2}
\end{aligned}
$$

where we have defined

$$
\begin{aligned}
Y_{1}= & 8 \eta^{2} l_{0} q^{2}+4 \eta \mu q^{2}-8 \eta^{2} l_{0}^{2} q^{3}+4 \mu b l_{0}^{2} q^{3}+4 \mu b q-4 \mu b q \cosh ^{2}\left(l_{0} q\right)-4 \mu b l_{0} q^{2} \\
& -2 \mu^{2} l_{0} q^{4}+2 \mu^{2} l_{0}^{2} q^{5}-8 b \eta \cosh ^{2}\left(l_{0} q\right)-8 \eta^{2} q \cosh ^{2}\left(l_{0} q\right)-2 \mu^{2} q^{3} \cosh \left(l_{0} q\right) \sinh \left(l_{0} q\right) \\
& -8 \eta b \cosh \left(l_{0} q\right) \sinh \left(l_{0} q\right)-2 \mu^{2} q^{3} \cosh ^{2}\left(l_{0} q\right)+2 \mu^{2} q^{3}-4 \mu b q \cosh \left(l_{0} q\right) \sinh \left(l_{0} q\right) \\
& -8 \eta \mu q^{2} \cosh \left(l_{0} q\right) \sinh \left(l_{0} q\right)+4 \eta b-8 \eta^{2} q \sinh \left(l_{0} q\right) \cosh \left(l_{0} q\right) \\
& -8 \eta \mu q^{2} \cosh ^{2}\left(l_{0} q\right) \\
Y_{2}= & \left(2 b \eta+4 \eta^{2} l_{0} q^{2}-4 \eta^{2} l_{0}^{2} q^{3}+\mu^{2} q^{3}+2 \eta \mu q^{2}+2 \mu b l_{0}^{2} q^{3}-2 \mu b l_{0} q^{2}\right. \\
& \left.+2 \mu b q+\mu^{2} l_{0}^{2} q^{5}-\mu^{2} l_{0} q^{4}\right) 2 \sinh \left(l_{0} q\right) \\
& +\left(2 b \eta-4 \eta^{2} l_{0} q^{2}+4 \eta^{2} q+4 \eta^{2} l_{0}^{2} q^{3}+2 \eta \mu q^{2}-2 \mu b l_{0}^{2} q^{3}+2 \mu b l_{0} q^{2}\right. \\
& \left.-\mu^{2} l_{0}^{2} q^{5}+\mu^{2} l_{0} q^{4}\right) 2 \cosh \left(l_{0} q\right)
\end{aligned}
$$

Having obtained the matrix of kinetic coefficients, we can now calculate the dynamical correlation functions. A formal solution to the relaxational dynamics, equation (12), is $\left(h_{q}, \rho_{q}, \vec{\rho}_{q}\right)(t)$ $=\exp (-\boldsymbol{\Gamma} \cdot \mathbf{E} t)\left(h_{q}, \rho_{q}, \bar{\rho}_{q}\right)(0)$. After diagonalization, one obtains the relaxation times of the various modes as the inverse values of the eigenvalues $\gamma_{\imath}$ of $\Gamma \cdot \mathbf{E}$.

The time-dependent correlation functions can be obtained from the corresponding eigenvectors $\mathbf{g}_{2}$. The matrix $\mathbf{U}=\left[\mathbf{g}_{1} \mathbf{g}_{2} \mathbf{g}_{3}\right]$ with matrix elements $U_{2 \jmath}$ diagonalizes $\boldsymbol{\Gamma} \cdot \mathbf{E}$. The dynamical correlation matrix $\mathbf{G}(t)$ is defined by

$\mathbf{G}(t) \equiv\left\langle\left(\begin{array}{c}h_{q} \\ \rho_{q} \\ \bar{\rho}_{q}\end{array}\right)(t)\left(h_{q}, \rho_{q}, \bar{\rho}_{q}\right)^{*}(0)\right\rangle=e^{-\boldsymbol{\Gamma} \cdot \mathbf{E} t} \mathbf{G}_{\mathbf{0}}=\mathbf{U} \exp \left(-\left[\begin{array}{ccc}\gamma_{1} & 0 & 0 \\ 0 & \gamma_{2} & 0 \\ 0 & 0 & \gamma_{3}\end{array}\right] t\right) \mathbf{U}^{-1} \mathbf{G}_{\mathbf{o}}$

In order to obtain the static correlation functions for $t \rightarrow 0$ we have used the fluctuation dissipation - theorem $\mathbf{G}(t=0)=\mathbf{G}_{0}=\left(k_{\mathrm{B}} T / L_{x} L_{y}\right) \mathbf{E}^{-1}$ For the matrix elements of $\mathbf{G}(t)$, equation (35) reads

$$
\begin{aligned}
\mathbf{G}_{\jmath k}(t) & =\sum_{\imath} \mathrm{e}^{-\gamma_{\imath} t} U_{\jmath 2} \sum_{m}\left(\mathbf{U}^{-1}\right)_{\imath m}\left(\mathbf{G}_{0}\right)_{m k} \\
& \equiv \sum_{\mathbf{2}} \mathrm{e}^{-\gamma_{\imath} t} A_{2}^{\jmath k}\left(\mathbf{G}_{\mathbf{0}}\right)_{3 k}
\end{aligned}
$$

where the condition $\mathbf{G}(t=0)=\mathbf{G}_{0}$ is equivalent to $\sum_{2} A_{2}^{j k}=1$. Here, the indices $\{1,2,3\}$ correspond to $\{h, \rho, \bar{\rho}\}$, i.e $\left\langle h_{q}(t) h_{q}^{*}(0)\right\rangle=\mathbf{G}_{11}(t)$, and so on. For convenience, we use $A_{\imath}^{h}$ instead of $A_{2}^{11}$, and $A_{\imath}^{\rho}$ for $A_{\imath}^{22}$.

\section{Appendix B.}

\section{Reynolds numbers and coupling to $\bar{\rho}$.}

The Reynolds number, Re, is a measure for the relevance of inertial and advective terms in the Navier-Stokes equation as compared to the friction term. For decaying plane waves it can be 
estimated by replacing the spacial derivatives by the wave-vector $q$, and the time derivative by the relaxation rate $\gamma$, leading to $\operatorname{Re}=\rho \gamma / \eta q^{2}$. Calculating the Reynolds number for the two slowest modes $\gamma_{1,2}$ gives $\operatorname{Re}_{1,2} \ll 1$, thus confirming the validity of the Stokes approximation, (Eq. (8)).

The coupling of $h$ and $\rho$ to $\bar{\rho}$, expressed in the amplitudes of the correlation function $A_{3}^{h}$ and $A_{\mathbf{3}}^{\rho}$, turns out to be negligible for all reasonable parameter values. These amplitudes are always less than $10^{-5}$ for the examples discussed in section 5 . We conclude that the error we made by treating also this mode within the Stokes approximation should not be harmful to our results. For the $\bar{\rho}$-mode, we find $\gamma_{3} \approx 2 k l q^{2} / \eta$ for small $q$, and $\gamma_{3} \approx k q /(2 \eta+\mu q)$, the result obtainable analytically for the free bilayer as well, for large $q$. However, these results are non-physical, since the Reynolds number for this mode, estimated as $\operatorname{Re}_{3}=\rho \gamma_{3} / \eta q^{2}$, turns out to be bigger that 1. A proper treatment of this mode would then reveal oscillatory "sound" modes within the membrane [21].

\section{Appendix C.}

\section{Measurement of correlations in a linear strip.}

In experiments using video microscopy, membrane displacements are frequently measured in a finite strip of length $L$ parallel to $x=0$, averaging over the width $B$ of the strip [32,9]. $B$ will most often be determined by the optical resolution of the microscope. Thus the observed quantity is

$$
H(x)=\frac{1}{B} \int_{-B / 2}^{B / 2} h(x, y) \mathrm{d} y .
$$

Defining the Fourier transform as $H(x)=\sum_{q_{x}} H_{q_{x}} \exp \left(i q_{x} x\right)$, one obtains from equation (38)

$$
\left\langle H_{q_{x}}^{2}\right\rangle=\sum_{q_{y}}\left\langle h_{\left(q_{x}, q_{y}\right)}^{2}\right\rangle \frac{4 \sin ^{2}\left(q_{y} B / 2\right)}{q_{y}^{2} B^{2}} \equiv \sum_{q_{y}}\left\langle h_{\left(q_{x}, q_{y}\right)}^{2}\right\rangle K_{s}\left(q_{y}\right)
$$

for the static height correlation function, where the correction factor $K_{s}\left(q_{y}\right)$ arises from the Fourier-transform of the finite strip. For $q_{y} \ll B^{-1}$, this factor reduces to unity. Projection on the strip is then equivalent to integration over all possible values of the component of the wave-vector perpendicular to the strip.

For an evaluation, we replace the sum by an integral, $\sum_{q_{y}} \rightarrow\left(L_{y} / 2 \pi\right) \int_{-\infty}^{\infty} \mathrm{d} q_{y}$. Using equation (7) for $\left\langle h_{\left(q_{x}, q_{y}\right)}^{2}\right\rangle$ we obtain in the limit $B \rightarrow 0$

$$
\left\langle H_{q_{x}}^{2}\right\rangle= \begin{cases}\frac{k_{\mathrm{B}} T}{L_{x}} \frac{\left(\kappa^{1 / 4} \sin \left(\frac{1}{2} \arccos (C)\right)\right.}{\left(\Omega+\Sigma q_{x}^{2}+\kappa q_{x}^{4}\right)^{1 / 4} \sqrt{-\Sigma^{2}+4 \kappa \Omega}}, \Sigma^{2}<4 \kappa \Omega \\ \frac{k_{\mathrm{B}} T}{L_{x} \sqrt{2 \Sigma^{2} / \kappa-8 \Omega}}\left(-\frac{\sqrt{\mid D^{+1}}}{D^{+}}+\frac{\sqrt{\mid D^{-1}}}{D^{-}}\right) & , \Sigma^{2}>4 \kappa \Omega,\end{cases}
$$

with

$$
C \equiv \frac{\Sigma+2 \kappa q_{x}^{2}}{2 \sqrt{\kappa} \sqrt{\Omega+\Sigma q_{x}^{2}+\kappa q_{x}^{4}}}
$$

and

$$
D^{ \pm} \equiv \Sigma+2 \kappa q_{x}^{2} \pm \sqrt{\Sigma^{2}-4 \kappa \Omega} \text {. }
$$


In the special case without a potential, $\Omega=0$, this expression has been previously derived by Mutz and Helfrich [32]. For small $q_{x}$, the function $\left\langle H_{q_{x}}^{2}\right\rangle$ is constant. For $\xi_{\Sigma}^{-1} \ll q_{x} \ll \xi_{\kappa}^{-1}$ it behaves as $\left(\sigma q_{x}\right)^{-1}$, and for $q \gg \xi_{\kappa}^{-1}$ it behaves as $\left(\kappa q_{x}\right)^{-3}$

The expression for the dynamical correlation function in the same geometry yields

$$
\left\langle H_{q_{x}}(t) H_{q_{x}}^{*}(0)\right\rangle=\frac{L}{2 \pi} \int \mathrm{d} q_{y}\left\langle h_{\left(q_{x}, q_{y}\right)}(t) h_{\left(q_{x}, q_{y}\right)}^{*}(0)\right\rangle=\frac{L}{2 \pi} \int \mathrm{d} q_{y}\left\langle h_{\left(q_{x}, q_{y}\right)}^{2}\right\rangle_{0} \sum_{\imath} A_{\imath}^{h} \mathrm{e}^{-\gamma_{x}\left(q_{x}, q_{y}\right) t}
$$

which has to be evaluated numerically. Thus, for every particular wave-vector $q_{x}$, a superposition of relaxation rates contributes to the correlation function.

As an example, we have performed the $q_{y}$-integration of the dynamical correlation function, equation (43) for the "classical" case of a free membrane without internal degrees of freedom. For $u \equiv \kappa q_{x}^{3} t / 4 \eta \ll 1$, the result is governed by the static correlations on the strip, $\left\langle H_{q_{x}}(t) H_{q_{x}}^{*}(0)\right\rangle \sim t \mathrm{e}^{-u} / 4 \eta u=\mathrm{e}^{-u} /\left(\kappa q_{x}^{3}\right)$. However, for $u \gg 1$, we obtain $\left\langle H_{q_{x}}(t) H_{q_{x}}^{*}(0)\right\rangle \sim$ $t \mathrm{e}^{-u} / 4 \eta u^{3 / 2}=2 \eta^{1 / 2} \mathrm{e}^{-u} / t^{1 / 2}\left(\kappa q_{x}^{3}\right)^{3 / 2}$, i.e. the correlations on the strip fall off faster with $q_{x}$ than in $\left\langle h\left(q_{x}, q_{y}=0, t\right) h\left(q_{x}, q_{y}=0,0\right)\right\rangle$.

\section{Appendix D.}

\section{Extensions of the model.}

In this section, we want to discuss briefly some simple extensions of the model applying to somewhat modified physical situations. So far, we have assumed a smooth solid wall with no-slip boundary condition. If the interface between substrate and adjacent fluid is coated by another lipid monolayer or bilayer, as is also the case for strongly adhering "pancake" vesicles (see example 2), it may be necessary to allow for a finite slip at $z=0$. This is done by introducing a friction term $\propto \tilde{b} v_{x}(z=0)$ with a phenomenological friction coefficient $\tilde{b}$ in the force-balance at the substrate that replaces the condition $v_{x}(z=0)=0$. It turns out that, for small $q$, this leads to a correction factor $\left(4+\tilde{b} l_{0} / \eta\right) /\left(1+\tilde{b} l_{0} / \eta\right)$ in the damping rate $(18)$ of the undulation mode. The $q^{2}$ - dependency of the damping, however, remains valid, since the slip does not change the conservation of volume between membrane and substrate. For coating with a bilayer, we may use $\tilde{b} \simeq b=10^{8} \mathrm{~J} \mathrm{~s} / \mathrm{m}^{4}$, which leads for $l_{0} \simeq 50 \mathrm{~nm}$ to $\tilde{b} l_{0} / \eta \simeq 5 \times 10^{3}$. In this case, the correction due to the slip is negligible. In the low-friction regime, that is for $\tilde{b} \ll l_{0} / \eta$, the correction can reach a factor of 4 .

In experiments, very often multilamellar vesicles are observed. In the case of adhesion, they may be described as a stack of membranes near a wall separated by small amounts of water [33]. Thus, for small $q$, the flow fields of the membranes affect each other leading to a modification of the damping rates. As a simple example, we consider two membranes without internal degrees of freedom as discussed in section 4 . Their distances to the wall will be denoted by $l_{1}$ and $l_{2}$, respectively, with a mean intermembrane distance $\Delta l \ll\left\{l_{1}, l_{2}\right\}$. We can expand the membrane-membrane interaction potential $V_{\mathrm{m}}\left(l_{2}-l_{1}\right)$ about $\Delta l$, with $\Omega_{\mathrm{m}} \equiv \mathrm{d}^{2} V_{\mathrm{m}} /\left.\mathrm{d}\left(l_{2}-l_{1}\right)^{2}\right|_{l_{2}-l_{2}=\Delta l}$. For $q \ll \xi_{\Sigma}^{-1}$, the two modes for two independent membranes with $\gamma \approx \Omega l_{\imath}^{3} q^{2} / 12 \eta$ (see Eq. $(21) ; i=1,2$ ) are replaced by two collective modes. The faster mode has essentially twice the undulation energy of a single membrane, while the second one, the "peristaltic" mode [3], can become very slow with

$$
\gamma_{1} \approx \frac{\Omega_{\mathrm{m}}(\Delta l)^{3} q^{2}}{12 \eta}
$$


since the length $l_{2}$ is replaced by $\Delta l$. Both modes lead to appreciable height fluctuations and should be visible in optical experiments.

\section{References}

[1] Lipowsky R., Nature 349 (1991) 475.

[2] The Structure and Conformation of Amphiphilic Membranes, Vol. 66 of Springer Proceedings in Physics, R. Lipowsky, D. Richter and K. Kremer Eds. (Springer, Berlin, 1991).

[3] Brochard F. and Lennon J., J. Phys. France 36 (1975) 1035;

Frey E. and Nelson D.R., J. Phys. I France 1 (1991) 1715.

[4] Engelhardt H., Duwe H. and Sackmann E., J. Phys. Lett. France 46 (1985) L-395.

[5] Bivas I., Hanusse P., Bothorel P., Lalanne J. and Aguerre-Chariol O., J. Phys. France 48 (1987) 855.

[6] Duwe H., Käs J. and Sackmann E., J. Phys. France 51 (1990) 945.

[7] Zilker A., Engelhardt H. and Sackmann E., J. Phys. France 48 (1987) 2139.

[8] Rädler J. and Sackmann E., J. Phys. II France 3 (1993) 727.

[9] Rädler J., Ph. D. thesis, TU-München (1993).

[10] Helfrich W., Z. Naturforsch. 28c (1973) 693.

[11] Seifert U., Phys. Rev. E 49 (1994) 3124.

[12] Seifert U. and Langer S., Biophys. Chem. 49 (1994) 13.

[13] Helfrich W. and Servuss R.-M., Il Nuovo Cimento 3D (1984) 137.

[14] Milner S. and Safran S., Phys. Rev. A 36 (1987) 4371.

[15] Evans E., Yeung A., Waugh R. and Song J., The Structure and Conformation of Amphiphilic Membranes, Vol. 66 of Springer Proceedings in Physics, R. Lipowsky, D. Richter and K. Kremer Eds. (Springer, Berlin, 1991) pp. 148-153.

[16] Seifert U., Miao L., Döbereiner H.-G. and Wortis M., The Structure and Conformation of Amphiphilic Membranes, Vol. 66 of Springer Proceedings in Physics, R. Lipowsky, D. Richter and K. Kremer Eds. (Springer, Berlin, 1991) pp. 93-96.

[17] Miao L., Seifert U., Wortis M. and Döbereiner H.-G., Phys. Rev. E, in press.

[18] Wiese W., Harbich W. and Helfrich W., J. Phys.: Cond. Matter 4 (1992) 1647.

[19] Waugh R., Song J., Svetina S. and Zeks B., Biophys. J. 61 (1992) 974.

[20] Fischer T., Biophys. J. 63 (1992) 1328.

[21] Seifert U. and Langer S., Europhys. Lett. 23 (1993) 71.

[22] Lipowsky R. and Leibler S., Phys. Rev. Lett. 56 (1986) 2541.

[23] The modes of an interface with dominating tension are discussed in A. Ammann, U. Seifert, and R. Lipowsky, to be published.

[24] Merkel R., Sackmann E. and Evans E., J. Phys. France 50 (1989) 1535.

[25] The permeability coefficient of lipid bilayer membranes is known to be of the order of $P=10^{-5}$ $\mathrm{m} / \mathrm{s}$ [34]. Converting the hydrostatic pressures to the concentration difference of a solute with the same osmotic pressure, we obtain a net flux of solvent through the membrane $\mathrm{d} Q / \mathrm{d} t=$ $P \Delta p / R T \approx 10^{-14} \mathrm{~kg} / \mathrm{m}^{2} \mathrm{~s}$. This is equivalent to a relative velocity of membrane and solvent of $10^{-17} \mathrm{~m} / \mathrm{s}$ which is totally negligible in comparison with typical membrane velocities of the order $\gamma h \approx 10^{-7} \mathrm{~m} / \mathrm{s}$. (A similar argument can be found in Youhei F., Physica A 203 (1994) 214) If transient pores are created dynamically by shear forces, one has to replace equation (10) by two seperate force balances for the membrane and the solvent at $l_{0}$. In the simplest approximation, this gives rise to a $q$-independent term in $\gamma_{s}$. Only for much higher permeabilities than measured statically one expects measurable effects. 
[26] This definition of $q_{1}^{f}$ differs from the one given previously in reference [21] by a factor $\kappa / \bar{\kappa}$ for the sake of better consistency with the respective definition for the case of a bound bilayer.

[27] With $q_{2} \simeq 10^{9} \mathrm{~m}^{-1}$, this crossover is of the order of the thickness of the bilayer, where the continuum description may break down.

[28] Pfeiffer W., König S., Legrand J., Bayerl T., Richter D. and Sackmann E., Europhys. Lett. 23 (1993) 457.

[29] Israelachvili J., Intermolecular and Surface Forces, 2nd Ed. (Academic Press, London, 1991).

[30] Evans E. and Needham D., J. Phys. Chem. 91 (1987) 4219.

[31] Char B.W., Geddes K.O., Gonnet G.H., Leong B.L., Monagan M.B., Watt S.M., Maple V Reference Manual (Springer, Berlin, 1991).

[32] Mutz M. and Helfrich W., J. Phys. France 51 (1990) 991.

[33] The unbinding of stacks of membranes has recently been discussed by Netz R.R. and Lipowsky R., Phys. Rev. Lett. 71 (1993) 3596; and by Lipowsky R., J. Phys.: Cond. Matter, in press.

[34] Sackmann E., Biophysics, W. Hoppe, W. Lohmann, H. Markl and H. Ziegler Eds. (Springer, Berlin, 1983) pp. 425-457. 